\title{
Pevista
}

ISSN-L: 2215-4728

DOI: http://dx.doi.org/10.15359/rp.22.3

http://www.revistas.una.ac.cr/perspectivas

N. ${ }^{\circ} 22$. Enero-Junio, $2021 \cdot$ pp. 1-16

\section{Los Derechos Humanos y su potencial desde la enseñanza de los Estudios Sociales \\ y Educación Cívica en la Universidad Nacional de Costa Rica: un estudio desde las propuestas de siete cursos de su plan de estudios}

\author{
Human Rights and its Potential from the Teaching of \\ Social Studies and Civic Education at the National \\ University of Costa Rica: A Study from the Proposals \\ of Seven Courses of its Curriculum
}

\author{
Roberto Granado Porras* \\ Lode Elena Cascante Gómez*
}

Fecha de recepción: 15/02/2021 • Fecha de aceptación: 20/03/2021

Resumen: El trabajo muestra los resultados de un análisis bibliográfico para fortalecer el tema de los derechos humanos en el bachillerato en la Enseñanza de los Estudios Sociales y Educación Cívica (EESEC), en la Universidad Nacional de Costa Rica. Para
Abstract: The present work shows the results of a bibliographic investigation that analyzes the possibilities of transversely strengthening the Human Rights issue from the Baccalaureate in the teaching of Social Studies and Civic Education (EESEC) at the

* Costarricense. Máster en Historia Aplicada y Máster en Tecnología e Innovación Educativa por la Universidad Nacional de Costa Rica (UNA). Profesor e investigador en la Escuela de Historia de la Universidad Nacional (UNA), Sede Central «Omar Dengo», Heredia, Costa Rica. roberto.granados.porras@ una.cr. ORCID: https://orcid.org/0000-0003-3460-5393.

** Costarricense. Máster en Pedagogía de la Diversidad por la Universidad Nacional de Costa Rica (UNA). Profesora e investigadora en la División de Educología de la Universidad Nacional (UNA), Sede Central «Omar Dengo», Heredia, Costa Rica. lode.cascante.gomez@una.cr. ORCID: https://orcid.org/0000-0002-1281-3821. 
lo cual se analizó el potencial de siete cursos de la malla curricular, del plan de estudios de 2017, y cómo se puede fortalecer la temática desde el desarrollo teórico y metodológico de estos cursos. La pesquisa se planteó desde un enfoque naturalista, por lo tanto, se está frente a un trabajo cualitativo desde una investigación básica de corte transversal. El principal resultado evidenciado en ella es que el planteamiento curricular del bachillerato en la EESEC tiene la posibilidad de convertirse en un referente para fortalecer los derechos humanos y su enfoque, dentro de una sociedad diversa y con tendencia a la exclusión.

Palabras claves: Derechos Humanos; educación; Educación Cívica; Estudios Sociales; planteamiento curricular; Costa Rica.
National University of Costa Rica.For which, the potential of seven courses was analyzed, from the curriculum that was developed in 2017, and how the theme can be strengthened from the theoretical and methodological development of these courses, emphasizing on increasing education as a Human Right. The research was raised from a naturalistic approach; therefore, it is facing a qualitative work from a basic cross-sectional research. The main result evidenced in the research is that the high school curricular approach at EESEC has the possibility of becoming a reference to strengthen Human Rights and its approach within a diverse society with a tendency to exclusion.

Keywords: Civic Education; curriculum planning; education; Human Rights; Social Studies; Costa Rica.

\section{Introducción}

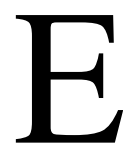
sta investigación tomará la formación inicial docente en la Enseñanza de los Estudios Sociales y Educación Cívica (EESEC) en la Universidad Nacional de Costa Rica, dentro de una propuesta para fortalecer la educación como un derecho humano. Se seleccionó este campo de la enseñanza, porque en su malla curricular existen diferentes temáticas que se dedican al estudio de los derechos humanos o a fortalecerlos como ejes transversales en sus diferentes cursos (Araya $e t$ al., 2017). Pero, haciendo alusión a la necesidad que el futuro profesorado se comprometa a robustecer este tema, más allá de prepararse para impartir un contenido en sus próximos contextos laborales.

El objetivo principal del presente artículo es analizar las principales metodologías que se pueden implementar desde la formación inicial docente en la EESEC, para mejorar la enseñanza de los derechos humanos como derecho universal. Desde esta propuesta, se pretende establecer el análisis de la educación de forma global y cómo en la actualidad, se convierte en un derecho humano. En los objetivos específicos se 
plantea revisar las propuestas curriculares de la EESEC para puntualizar en el análisis de los cursos que abordan la enseñanza de los derechos humanos, proponer diferentes estrategias metodológicas para fortalecer su enseñanza y profundizar en el tema de la educación como un derecho universal desde la EESEC.

Dentro de los antecedentes para la propuesta se cuenta con el trabajo de Barrios (2020) «La educación en derechos humanos y el currículo oculto: consideraciones teórico-prácticas sobre el cotidiano escolar brasileño» que enfatiza en las relaciones socioconstructivistas donde intervienen una serie de elementos y directrices que permiten reflexionar en el currículo oculto para la implementación de la educación en derechos humanos. El trabajo plantea que se debe desarrollar una «pedagogía participativa, que haga de la EDH [Educación en Derechos Humanos] un elemento relevante para los alumnos, y también una formación docente que parta del análisis de las prácticas pedagógicas y de las acciones programáticas de la $\mathrm{EDH}$ (Barrios, 2020, p. 7).

Uno de los principales problemas que señala la autora anterior, es que la formación en derechos humanos queda reducida a un segundo plano, ante la transmisión unilateral de contenidos (Barrios, 2020). Situación que se considera comprometedora, ante acciones mundiales que se direccionan a una exclusión social mayor. La formación inicial docente, en EESEC, se considera como un espacio de reflexión que posibilita el fortalecimiento de los derechos humanos desde los primeros años de instrucción. El perfil de un profesorado comprometido, desde el inicio de su carrera, con la imperante necesidad de educar a partir de los principios de los derechos humanos como un valor universal y acorde a su época, cada vez más diversa, facilitará el desarrollo de prácticas y acciones mucho más equitativas y humanas desde el aula. Se requiere de una persona docente con el conocimiento y con dominio en prácticas de aula, donde los principios de los derechos humanos sean el eje de su mediación educativa, y, no por repetición de estrategias didácticas sin sentido pedagógico.

Otra investigación de González (2015) «Educación, Derechos Humanos y Shoá: Una Aproximación desde la experiencia teórico-práctica», particulariza en las experiencias vividas por las personas que fueron víctimas del holocausto. Su estudio lleva al estudiantado a profundizar 
en la importancia de una educación basada en derechos humanos. Este trabajo precisa cómo las formas de poder organizadas traspasan todas las libertades individuales, hasta llegar a cometer atrocidades como la Shoá (González, 2018). De igual forma, este es un contenido que se desarrolla desde la EESEC y con una adecuada mediación en Ciencias Sociales se pueden fortalecer las habilidades que lleven a una apropiada comprensión de lo que sería vivir en sociedades diversas.

Por último, en el trabajo de Cortéz (2011) «Los derechos humanos en las ciencias sociales: una perspectiva multidisciplinaria», se hace énfasis en los distintos materiales desarrollados desde las ciencias sociales para atender esta temática. Resalta que en muchos de los artículos escritos al respecto, se cuenta con una connotada ayuda didáctica, pues existen diferentes aristas que se han trabajado a partir de estas ciencias, las que permiten tener «una visión sintética de temas complejos» (Cortéz, 2011, p. 230). Toda esta discusión, en las ciencias sociales, es la que se debe acuerpar desde la EESEC, con el objetivo de profundizar en cuestiones que fortalezcan su enseñanza y, por ende, su aporte al perfil profesional de la persona graduada.

\section{Conceptualización teórica}

Para iniciar, se toma el concepto de la enseñanza de los Estudios Sociales y Educación Cívica, que se caracteriza por ser dinámico y cargado de complejidad. La EESEC se incluye dentro de una visión disciplinar que va más allá de asignaturas escolares. Su visión teórica se fortalece por medio de un cuerpo epistemológico en construcción que se apoya de la investigación académica, educativa y pedagógica.

En el plan de estudios de la Licenciatura de la Enseñanza de los Estudios Sociales y Educación Cívica de la Universidad Nacional se mencionó que la formación del profesorado debía responder a una sociedad en constante transformación, que aporte al desarrollo sociopolítico del país por medio de la obtención de un conocimiento y pensamiento crítico, que sea propositivo ante las dinámicas y problemáticas sociales (Bolaños, et al., 2014). Esta conceptualización no es diferente a la que se emplea en los planes del Bachillerato en Estudios Sociales, que hacen alusión a una EESEC multidisciplinar, en consonancia con las 
características que están presentes en la sociedad actual (López, 2018). De ahí que, una educación en derechos humanos necesita estar presente como un eje transversal, en la formación inicial docente.

Por su parte, Wayne Ross (2014) hace referencia a unos Estudios Sociales inclusivos, claves en el conocimiento social y, que, se caracterizan por una discusión permanente. Además, mencionó que desde inicios del siglo XX este campo de enseñanza se orientó a consolidar los valores y políticas del buen ciudadano. Situación que coincide, para el caso costarricense, con la EESEC, la cual se relaciona con la transmisión de valores y la historia oficialista, lo que se convierte en uno de los principales retos a trabajar en los contextos escolares.

Para ahondar en los derechos humanos, la mayoría de teorías sostienen que todas las personas tienen derechos que son inherentes a su propia condición humana. Es decir, que el «respeto hace posible construir y mantener sociedades que convivan en libertad, justicia y paz. Son los llamados derechos humanos» y son considerados como «el ideal común por el que todos los pueblos y naciones deben esforzarse» (Rodino, 2015, pp. 5-8). Para cualquier planteamiento teórico-conceptual que se tome, se establecen tres dimensiones que constituyen la complejidad de los derechos humanos: una dimensión axiológica o ética, que hace referencia al cuerpo de valores que se relacionan con la dignidad de la persona, la dimensión histórico-cultural, que explica el origen de los valores y las normas que se derivan de ellos y, la dimensión política-jurídica, que desarrolla el contenido legal de los derechos y que obliga al cumplimiento de estos en el nivel social (Rodino, 2015).

Cuando se requiere generar un proceso real y consciente de la importancia de los derechos humanos se debe considerar que su aceptación demanda de una reflexión valorativa sobre qué es el ser humano, una reflexión histórica que facilite crear la necesidad de la universalización y el rescate de las particularidades culturales. Sin entrar en disputas conceptuales, se puede considerar que estos son:

un conjunto de facultades o atribuciones histórico-culturales (derechos) reconocidos jurídicamente a toda persona, por el hecho de ser persona (derechos subjetivos), por las cuales ésta puede actuar para satisfacer unas necesidades o aspiraciones inherentes a su cualidad humana, y que 
tienen, por ser, una radical importancia para ella. (López-Barajas, 2013, p. 32)

Lo que se pretende con estos derechos es que el ser humano pueda proteger los intereses o aspiraciones que lo llevan a realizarse como persona, con el objetivo que pueda desarrollarse en el nivel comunitario con igualdad, dignidad y libertad. Ahora bien, la educación se convierte en un elemento potenciador y valorativo de estos derechos en el ámbito cultural y social. Como menciona el autor anterior, la educación se convierte en «la base ineludible y la condición necesaria, aunque no suficiente, para la promoción y vigencia de una cultura de los derechos humanos. Sólo una educación en los valores implícitos en esos derechos garantiza un desarrollo estable de los mismos» (López-Barajas, 2013, p. 41).

Por la particularidad de este trabajo se intenta robustecer la educación cual derecho humano, que desde la formación inicial en la EESEC se considera un contenido transversal a desarrollarse en cualquier temática. Es un elemento de especial atención que debe llevar a reflexiones profundas y que, en el nivel de contenidos se trabaje de forma cotidiana, en los distintos cursos ofrecidos en la Escuela. Cuando se habla de la educación como un derecho, esta afirmación tiene la particularidad que se le asignan fines específicos como «alcanzar el pleno desarrollo de la personalidad, el respeto a los derechos humanos y las libertades fundamentales, la comprensión, la tolerancia, y amistad entre naciones y los grupos humanos y promover el mantenimiento de la paz» (Rodino, 2015).

Desde la formación inicial en la EESEC al defender estos preceptos, posiblemente se estará trabajando en potenciar, a futuro, una sociedad más justa, que será promovida por un profesorado que llegará con una base sólida de formación en derechos humanos; al fortalecer los principios claves que orienten el trabajo docente, desligándose de conceptos espontáneos e inmediatistas que no generan fundamentos de orden ético y esencialmente humanos. Inclusive, cada persona pedagoga, tanto en formación como en ejercicio, debe tener claro que la educación es un derecho establecido en la Declaración Universal de Derechos Humanos, en su Artículo 26 y, por lo tanto, se debería tener como objetivo la potenciación de esta regulación. Sobre todo, al considerar que en la actualidad la educación en los derechos humanos es una 
labor que debe ser prioridad en las personas docentes, pues, desde ella se crean conocimientos que favorecen el desarrollo de actitudes activas para defender sus principios y, a la vez, trabajar de forma colaborativa para llegar a la construcción de una sociedad más equitativa y justa.

De acuerdo con Boronat y Ruiz (2013), la formación inicial del profesorado cuenta con algunas implicaciones como:

- La adquisición de conocimientos relativos a los derechos humanos, de manera metódica y progresiva a lo largo de todo el proceso formativo.

- La educación de derechos humanos afecta al conjunto de las áreas y disciplinas que integran el curriculum.

- La transversalidad y más concretamente la educación moral y cívica, es el espacio más adecuado para profundizar en esta formación, ya que la educación moral y cívica se basa en el desarrollo de principios con valor universal: dignidad del individuo, respeto a los demás, tolerancia, solidaridad, justicia que implica la tarea del educar. (p. 220)

Uno de los principales desafíos con los que cuenta el profesorado de la EESEC es educar para una ciudadanía democrática en el siglo $\mathrm{XXI}$, tomando en cuenta las diferentes transformaciones sociales que se enfrentan en la actualidad, tanto en el nivel costarricense como regional. Los cambios económicos, tecnológicos, de comunicación, políticos e ideológicos hacen que, ejercer la profesión docente sea un reto y deba desarrollarse con metodologías adecuadas para buscar la mayor equidad posible desde los contextos educativos y que la educación sea ese derecho humano protegido por un código ético, con propuestas curriculares que buscan la equidad de todos los contextos sociales (CEPPE, 2015).

Por último, el artículo es de corte bibliográfico y centra su atención en el análisis de una serie de cursos del Bachillerato en la Enseñanza de los Estudios Sociales y Educación Cívica (BEESEC) y su potencialidad para trabajarlos a partir de los derechos humanos. Como primer paso, se realizó una revisión de artículos y libros provenientes de las bases de datos de la Universidad Nacional como elibro y EBSCOhost, con el objetivo de recopilar información atinente a la temática. En segundo lugar, se desarrolló una exploración de libros y materiales en físico que 
complementaron el insumo digital. Por último, se analizó la malla curricular del Bachillerato en la Enseñanza de los Estudios Sociales y Educación Cívica (BEESEC) del 2017, para reconstruir el potencial de los cursos que se citan en esta investigación.

\section{Análisis de la malla curricular desde los derechos humanos}

Los resultados presentan el análisis de siete cursos de la malla curricular del bachillerato en EESEC y su aporte al tema de indagación en este ámbito educativo. En primera instancia, el plan del bachillerato de la EESEC, que se formuló en el año 2017, inició con un enfoque más diverso y centró su atención en la fundamentación de un objeto de estudio, en función de un marco conceptual y metodológico que potenció el desarrollo de capacidades, destrezas y habilidades propias de la enseñanza de los Estudios Sociales y la Educación Cívica. En este caso, se podría hablar de aquellas que basaron su atención en los derechos humanos y cómo estos podrían estar presentes de forma transversal en cada una de las temáticas que se desarrollan, tomando en cuenta que la EESEC presenta un enfoque amplio y multidisciplinario que incita a la reflexión constante.

De igual forma, dentro de la propuesta curricular del 2017, se incluyó la ética como un eje central del plan estudios (Araya, et al., 2017) para el desarrollo de los Derechos Humanos, dado que el conocimiento axiológico guía el ordenamiento jurídico que fortalece la propuesta base a la que apuntan este tipo de derechos (Rein, 2013; Rodino, 2015). La enseñanza de los EESEC plantea que el estudiantado cuente con una serie de retos cognitivos que lo lleven a reflexiones profundas, consientes y, sobre todo, comprometidas con su desarrollo y perfil profesional, por eso se requiere impulsar discusiones que retomen los derechos humanos desde las propuestas didácticas que se plantean en los ambientes de clase.

De acuerdo con el colectivo de investigación, que trabajó en la malla curricular del 2017, el mayor reto fue desarrollar un plan de estudios que fuera más allá de los contenidos históricos, geográficos y pedagógicos, con el objetivo de fortalecer el 
... aprendizaje del pensamiento crítico y a las capacidades, habilidades y destrezas propias de cada disciplina, además de integrar la ética profesional, la multidisciplinariedad y de formar un docente capaz de responder a los retos de la sociedad actual y de incidir en una sociedad democrática, y que cada curso dé cuenta de esa necesidad de incidir en las transformaciones sociales, políticas y culturales de nuestra época. (Araya et al., 2017)

Lo anterior refleja lo que se pretende en esta investigación en relación con la formación inicial docente. Se piensa en una persona que vaya más allá de ser un didacta sin sentido pedagógico del proceso, una que tenga como objetivo fortalecer el desarrollo de la sociedad democrática, donde la justicia social sea el eje central. La EESEC pretende una formación multidisciplinaria desde la Ciencias Sociales, con solidez y la calidad requerida, para que se pueda contribuir significativamente en la «construcción de una sociedad democrática acorde con las transformaciones y demandas actuales de la sociedad costarricense» (Araya et al., 2017).

La EESEC procuran que, desde la formación inicial, se aspire a una sociedad más inclusiva, donde las futuras personas estudiantes conozcan, analicen y valoren los avances culturales de la humanidad con el objetivo de fortalecer la construcción de una convivencia armoniosa en medio de la diversidad social y de la reproducción del sistema democrático (Araya et al., 2017). Es decir que, cada una de las oportunidades de aprendizaje que se desarrollan desde la EESEC deben ser espacios de reflexión que se aprovechen, con el objetivo de promover la convivencia democrática e impulsar los derechos humanos (Rein, 2013; Rodino, 2015). Se requiere potenciar una persona docente que trascienda el espacio del aula, el ser dador de información; por una con conocimientos reales y sensibilidad social.

En cuanto al perfil de las personas graduadas en el bachillerato de la EESEC, se proponen tres habilidades actitudinales, en la que los derechos humanos son un eje central. Por ejemplo, se plantea la toma de conciencia como una persona comprometida para el desarrollo de experiencias de aprendizaje en perspectiva de transformación social, territorial y ambiental; se establece asumir, desde el quehacer académico, un 
rol protagónico de manera continua, en fusión con los variados contextos de aprendizaje; ser consciente y ejercer una ciudadanía participativa y proponente en el marco de los derechos humanos, la justicia y la equidad, en su quehacer profesional, a partir del reconocimiento de diversas problemáticas sociales, políticas y ambientales originadas a través del tiempo, en contextos particulares y globales; demostrar el compromiso crítico y consiente por socializar los saberes construidos, la conciencia territorial y la valoración de los diferentes paisajes sociales (Araya et al., 2017).

Hasta el momento se analizó cómo la fundamentación de la EESEC, puede fortalecer los derechos humanos. Ahora y, de acuerdo con la malla curricular del 2017, se verá la oportunidad que tienen algunos cursos para robustecer el tema de estudio desde su acción sustantiva. Por ejemplo, desde el curso de Metodología de la Investigación para la EESEC se propone la investigación social con todos sus componentes: tema, problema, objetivos, marco conceptual, marco teórico, estado de la cuestión, hipótesis, introducción, capítulos, conclusión, bibliografía. En él se puede incrementar la investigación de diferentes temáticas relacionadas con los derechos humanos, como el derecho al trabajo, la salud física, mental o al libre tránsito (Garay y Pérez, 2018). Este tipo de indagación no solo prepara al estudiantado en su formación académica, sino que le lleva a profundizar en temas que deberán ser consolidados cuando llegue a ejercer profesionalmente.

Dentro de la propuesta, otro curso que puede fomentar la temática es el denominado Desarrollo Humano y Teorías del Aprendizaje para la EESEC. Con este, se pretende potencializar el sentido del desarrollo humano, dentro de las teorías del aprendizaje, con el objetivo de incidir de forma positiva en la formación académica del estudiantado. Dentro del desarrollo humano se incorpora la estabilidad que debe tener cualquier persona en la sociedad, en la cual, los individuos deben trabajar por su bienestar y estabilidad emocional (Figueiredo et al., 2019; Rodríguez y Quintanilla, 2019). Es decir, que dentro del desarrollo humano también deben estar incorporados, de forma transversal, los enfoques de los derechos humanos. Lo anterior está mencionado en uno de los objetivos del curso, que propone desarrollar habilidades para la formación de profesionales de la educación críticos, reflexivos, transformadores de 
la realidad educativa desde una visión integral del ser humano (Araya et al., 2017).

Un curso transcendental para profundizar en el fortalecimiento de la EESEC es el denominado Teoría Social, en él se plantea el debate de las diferentes teorías sociales y sus tendencias desde la enseñanza. Permite un amplio espectro de análisis, puesto que toma el funcionalismo, el estructuralismo, el socialismo, el marxismo, el simbolismo, la semiótica, semiología, lingüística, teoría crítica y líneas teóricas contemporáneas (Araya et al., 2017). Por medio de análisis e investigaciones del estudiantado se pueden realizar comparaciones teóricas y analizar la evolución de los derechos humanos en de los disímiles períodos históricos y las diferentes teorías. La diversidad teórica podrá incitar a la discusión sobre las distintas ideologías y cómo estas colaboraron en el desarrollo de los derechos humanos (Matthews, 2014).

Siguiendo la línea de la inclusión social se planteó el curso de Educación para la Diversidad, la Inclusión y la Cohesión Social. Este tiene como objetivo sensibilizar al futuro profesorado para interactuar en sociedades totalmente diversas, donde el respeto es la base de toda relación en el aula. Uno de los ejes de discusión centrales es la autorreflexión y el análisis de las paradojas, dilemas y retos que exige la educación para la diversidad y la inclusión, en ella se consideran los marcos jurídicos, epistemológicos y filosóficos de la educación para la inclusión, la investigación educativa como proceso de acercamiento al abordaje y la comprensión de la diversidad en el aula (Araya et al., 2017). En este caso, el análisis de la diversidad también implica la reflexión en cuanto a los derechos humanos y cómo las políticas de diversidad cultural, sexual, política e ideológica son protegidos desde esta (Celorio, 2017).

Este curso busca desarrollar la diversidad de la cultura del aprendizaje, al otorgar importancia, desde los principios teóricos, a la diversidad humana como eje para conocer la interacción de las personas estudiantes en su entorno cultural inmediato, para comprender el hecho de que las personas viven en ambientes muy diversos y complejos, con diferentes privaciones, que inciden en sus procesos de aprendizaje. De ahí que, la diversidad humana y sus diferentes derechos constituyen ejes claves en la formación académica, no solo, desde lo teórico, sino desde el acercamiento con la cultura de aprendizaje. Lo precedente significa 
que se debe construir un tratamiento educativo transformado a la realidad de la diversidad cultural como un derecho humano, en la construcción de nuevas realidades sociales.

Los cursos de formación histórica también deben ser considerados como una plataforma para la discusión y fortalecimiento de los derechos humanos. Por ejemplo, Historia de América Latina y el Caribe II, centra su estudio en el período entre 1808 a 1914, etapa en la cual se inicia la disputa sobre la independencia y las libertades individuales que buscaron los países de la región. Principalmente, se ejercieron luchas como la abolición de la esclavitud, la propiedad privada, la independencia económica, las libertades políticas, la libre expresión, entre otras; que son consideradas como los derechos humanos de primera generación. Es decir, las temáticas propuestas sirven como aliciente para profundizar en la investigación y en el desarrollo de habilidades desde la enseñanza de la historia. Lo mismo sucede con el período histórico señalado, para el caso de Historia de Costa Rica II, que se ajusta a las mismas temáticas para el caso costarricense, donde tanto el profesorado como el estudiantado podrían proponer diferentes enfoques que se ajusten a los derechos humanos.

En cuanto a la Historia Contemporánea, se propone analizar, en forma crítica y comparada, los principales procesos históricos ocurridos en África, Europa y Asia durante los siglos XIX y XX. Este curso, sin duda llevará al análisis de lo que ocurrió en la Primera y Segunda Guerra Mundial y cómo sus consecuencias facilitaron la articulación de entidades que se dedican al resguardo de los derechos humanos, con el objetivo de que no se repitan retrocesos históricos como los surgidos en este período (Jurado, 2013; Loughlin, 2016). El curso, pretende un análisis global y llevar a prácticas reflexivas desde la dinámica planetaria.

Por último, y para los objetivos de esta investigación se encuentra el curso Estado, Sociedad y Ciudadanía que analiza la dinámica del desarrollo institucional costarricense desde una perspectiva comparada. Este se considera como uno de los más importantes para los derechos humanos, pues toma un análisis global de temas como las fuerzas vivas frente al poder, los movimientos sociales, étnicos, sexuales y ecológicos, la participación ciudadana, las luchas sociales frente a los intereses políticos y empresariales, los derechos humanos y las luchas por 
su inclusión, constitución y evolución, los adultos mayores frente a la exclusión social, la diversidad sexual, cultural y étnica, lo inmigrantes y los migrantes, entre otros (Araya, et al., 2017). Entonces, se está ante una propuesta de aprendizaje que toma los derechos humanos como una propuesta curricular central, que lleva a dinámicas y análisis que buscan analizar las diferentes luchas que la humanidad ha enfrentado para buscar una inclusión social (Pazos, et al., 2020).

Es importante mencionar que, la presente investigación fue un análisis bibliográfico de las posibilidades de enseñanza y fortalecimiento de los derechos humanos desde la EESEC. Como se analizó, el potencial que presenta este campo de la enseñanza se basa en que el futuro profesorado cuente con herramientas que lleven a la transformación de la EESEC, desde un enfoque más humano y participativo. A partir del planteamiento curricular en el nivel de la Universidad Nacional, los Estudios Sociales y la Educación Cívica cuentan con una propuesta inherente a los derechos humanos y como se analizó, el planteamiento de la malla curricular lo tiene presente y el perfil de salida proyecta un profesional comprometido con estos.

Dentro de la propuesta curricular analizada se puede profundizar en los derechos humanos de todas las generaciones, pues como se planteó, la mayoría de los cursos pueden abrir sus análisis a diferentes temáticas, más allá de las que se proponen en dicha malla. Pero, estará en la posibilidad y actitud de cada persona docente el acuerpar toda la propuesta de los derechos humanos, para fortalecerlos desde sus actividades académicas. Se apunta a la construcción de puentes sociales que deben ser fortalecidos desde la academia, con el objetivo de generar un profesorado más comprometido con las problemáticas sociales.

También, dentro de la propuesta no se debe olvidar que la educación, como un derecho humano universal, debe estar presente. Se está frente a una formación de futuros docentes, personas comprometidas con la educación más allá de una simple formación académica. Desde la EESEC se debe fomentar y hacerle ver al estudiantado que la educación es uno de los derechos humanos más importantes, uno de los mejores instrumentos de movilidad social con el que se cuenta y, que a pesar del control político que se ejerce sobre ella, está llena de oportunidades para las personas de escasos recursos. Por eso, se propone que dentro de la 
EESEC, el futuro profesorado potencie esta visión de la educación como una estrategia de movilidad social y participación ciudadana democrática, en una sociedad que enfrenta serios problemas de inclusión.

\section{Conclusiones}

Como se analizó, se está ante una de las temáticas más importantes dentro de una sociedad que cambia y obliga a replanteamientos sociales. Hace quizá un par de años, cuando se discutía sobre derechos humanos se creía que ya se había avanzado lo suficiente, pero se está enfrentando retrocesos en el nivel mundial, fundamentados en prácticas que buscan naturalizar su violación. Gobiernos autoritarios, violencia de género, violencia interracial, homofobia o xenofobia son problemáticas que toman fuerza en la actualidad. Más allá de haber superado esta oleada de conflictos, que en otrora eran comunes, las primeras décadas del nuevo milenio trajeron consigo retrocesos que parecían superados por la humanidad. En el caso costarricense, la oposición al matrimonio igualitario, el aborto, la xenofobia y el deterioro del Estado Social de Derecho golpean fuertemente a nuestra sociedad.

Por lo anterior, se tomaron los derechos humanos como una propuesta de potencialización desde la EESEC, dado las posibilidades con las que cuenta esta disciplina para robustecer la temática. Además, para trabajar con el futuro profesorado nuevas propuestas de inclusión, necesarias en tiempos conflictivos. Se propone que, desde la disciplina se trabaje la necesidad de potenciar la educación como un derecho universal que debe ser fortalecido desde los contextos sociales y áulicos.

De igual manera, se analizó la malla curricular de la Universidad Nacional de Costa Rica, dada la posibilidad que existe en sus cursos de aportar en el nivel nacional, para dar a entender que el tema de los derechos humanos no es algo acabado, sino que está en construcción cotidianamente. Claro está que no es una propuesta curricular perfecta, puesto que se admiten los errores que pueda contener, pero el objetivo es apoyar su crecimiento y hacer de los derechos humanos una característica central como lo menciona su perfil de salida. 


\section{Referencias}

Araya, I., Barrantes, G., Rostrán, M., González, F., López, M., Ramírez, J., Silva, M., \& Vargas, A. (2017). Plan de Estudios del Bachillerato en la Enseñanza de los Estudios Sociales y Educación Cïvica.

Barrios, A. (2020). La educación en derechos humanos y el currículo oculto: consideraciones teórico-prácticas sobre el cotidiano escolar brasileño. Educación, 29(56), 7-26. https://doi.org/10.18800/educacion.202001.001.

Bolaños, C., Ramírez, J., \& Sanabria, O. (2014). Plan de Estudios Licenciatura en la Enseñanza de los Estudios Sociales y Educación Cívica Modalidad: Bimodal.

Boronat, J., \& Ruiz, E. (2013). Una propuesta didáctica para la inclusión de los derechos del niño en el currículum de formación inicial del profesorado. En E. Lopez-Barajas (Ed.), Derechos Humanos y Educación (pp. 221-241). Universidad Nacional Estatal a Distancia (UNED). https://elibro.net.una.remotexs.co/es/lc/unacr/titulos/48385.

Celorio, M. (2017). Violencia biopolítica contra poblaciones de la diversidad sexual: homofobia, derechos humanos y ciudadanía precaria. El Cotidiano, (202), 17-29.

CEPPE. (2015). Educación UC profundiza la importancia de contenidos de Derechos Humanos en la formación de sus profesores. CEPPE · Centro de Estudios de Políticas y Prácticas En Educación.

Cortéz, É. (2011). Los derechos humanos en las ciencias sociales: una perspectiva multidisciplinaria. Norteamérica, 6(1), 219-228.

Figueiredo, G., Weihmüller, V., \& Orrillo, Y. (2019). The results of socio-educational projects on the human development of young people. Educacao and Realidade, 44(4), 1-23. https://doi.org/10.1590/2175-623687645.

Garay, C., \& Pérez, G. (2018). Los derechos humanos en Colombia: Acuerdo final de paz y su proyección en la política de seguridad y defensa. Revista Científica General José María Córdova, 16(23), 84-105. https://doi.org/10.21830/19006586.307.

González, L. (2018). Educación, Derechos Humanos y Shoá: Una Aproximación desde la experiencia teórico-práctica. Cuadernos Judaicos, (35), 87-105. https://doi.org/10.5354/0718-8749.2018.52017.

Jurado, R. (2013). Luces y sombras del origen de la onu y la Declaración Universal de Derechos Humanos. El Cotidiano, (180), 31-40. 
López-Barajas, E. (2013). Derechos humanos y educación. Universidad Nacional Estatal a Distancia (UNED). https://elibro.net.una.remotexs.co/es/ereader/unacr/48385.

López, M. (2018). La enseñanza de la HISTORIA en COSTA RICA: Un acercamiento desde los planes de la carrera de Estudios Sociales en la Universidad Nacional (1979-2005). Perspectivas, (16), 1-22. https://doi.org/10.15359/rp.16.2.

Loughlin, J. (2016). Human dignity: The foundation of human rights and religious freedom. Memoria y Civilizacion, 19, 313-343. https://doi.org/10.15581/001.19.313-343.

Matthews, R. (2014). Critical realism: A structural analysis. Politica Criminal, 9(17), 182-212. https://doi.org/10.4067/S0718-33992014000100006.

Pazos, L. S., Teixeira, C., \& Feregrino, J. (2020). Pensar Desde a América Latina Em Sociais Descoloniais : Explorando. RAE-Revista de Administração de Empresas, 60(2). https://doi.org/http://dx.doi.org/10.1590/S0034-759020200203.

Rein, T. (2013). Participación política de las mujeres: Aspectos de confluencia entre la ética y los derechos humanos. Acta Bioethica, 19(2), 219-228. https://doi.org/10.4067/s1726-569x2013000200006.

Rodino, A. (2015). Educación en derechos humanos para una ciudadanía democrática. EUNED.

Rodríguez, E., \& Quintanilla, A. (2019). Relación del ser humano-naturaleza: Desarrollo, adaptabilidad y posicionamiento hacia la búsqueda de bienestar subjetivo. Avances En Investigación Agropecuaria, 23(3), 7-22. http://ww.ucol.mx/revaia/portal/pdf/2019/sept/1.pdf.

Ross, W. (2014). The Social Studies Curriculum: Purposes, Problems, and Possibilities (E. Wayne Ross (ed.); Fourth Edi). State University of New York, Albany. 